IN3 Working Paper Series INTERNET INTERDISCIPLINARY INSTITUTE

\title{
Structural Reliability and Availability Analysis through Simulation
}

Angel A. Juan \& Jorge Simosa (ajuanp@uoc.edu, jdsimosa@mit.edu)

IN3-Open University of Catalonia, Spain

Javier Faulin (javier.faulin@unavarra.es)

Public University of Navarra, Spain

Arai Monteforte \& Harry Guo (\{arai.monteforte, harry.guo\}@reliasoft.com)

ReliaSoft Corporation, USA

\section{Working Paper}

Working Paper Series WP10-002

Research group: HAROSA KC

Research group coordinator: Angel

A. Juan (IN3-UOC)

Submitted in: 2010

Accepted in: 2010

Published in: 2011

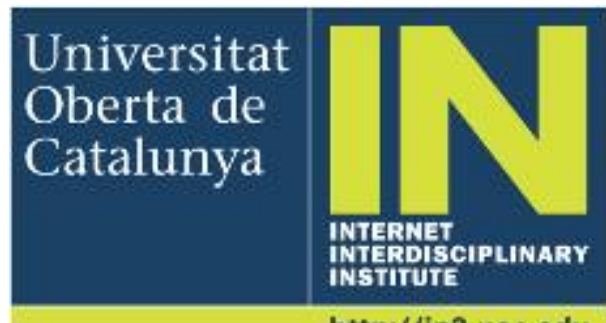




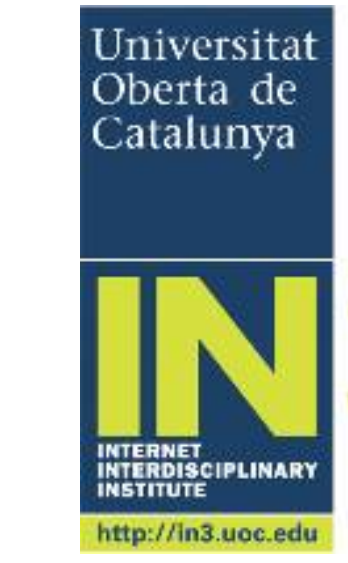

Internet Interdisciplinary Institute (IN3)

http://www.in3.uoc.edu

Edifici MediaTIC

c/ Roc Boronat, 117

08018 Barcelona

Espanya

Tel. 934505200

Universitat Oberta de Catalunya (UOC)

http://www.uoc.edu/

Av. Tibidabo, 39-43

08035 Barcelona

Espanya

Tel. 932532300

\section{(a)}

The texts published in this publication are - unless indicated otherwise - covered by the Creative Commons Spain Attribution-Non commercial-No derivative works 3.0 licence. You may copy, distribute, transmit and broadcast provided that you attribute it (authorship, publication name, publisher) in the manner specified by the author(s) or licensor(s).

The full text of the licence can be consulted here:

http://creativecommons.org/licenses/by-nc-nd/3.0/es/deed.en. 


\section{Table of contents}

Table of contents 3

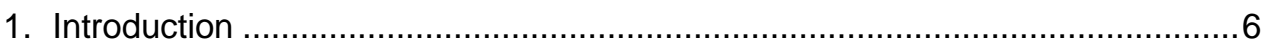

2. Structural Reliability and Existing Analysis Methods ............................... 7



4. Experiment 1: Structural Reliability ..................................................... 12

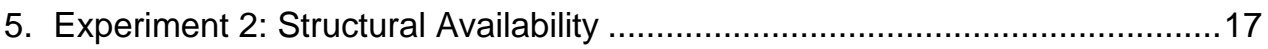

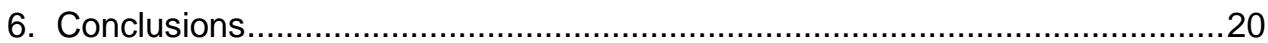

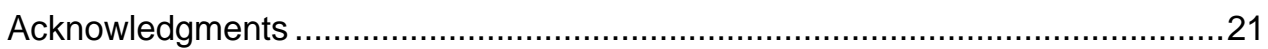

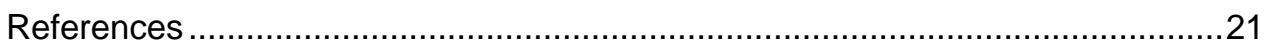

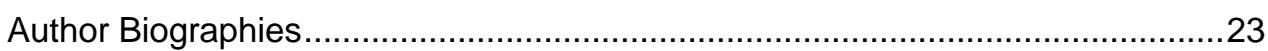

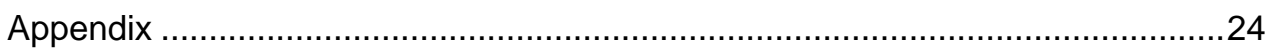




\section{Structural Reliability and Availability Analysis through Simulation}

Angel A. Juan \& Jorge Simosa (ajuanp@uoc.edu, jdsimosa@mit.edu) IN3-Open University of Catalonia, Spain

Javier Faulin (javier.faulin@unavarra.es)

Public University of Navarra, Spain

Arai Monteforte \& Harry Guo (\{arai.monteforte, harry.guo\}@reliasoft.com) ReliaSoft Corporation, USA

Recommended citation:

JUAN, Angel; SIMOSA, Jorge; FAULIN, Javier; MONTEFORTE, Arai; GUO, Harry (2011). "Structural Reliability and Availability Analysis through Simulation" [online working paper]. (Working Paper Series; WP 10-002). IN3 Working Paper Series. IN3 (UOC). [Accessed: dd/mm/yy].

<http://in3wps.uoc.edu/ojs/index.php/in3-working-paper-series/article/view/n10juan/n10-juan> 


\begin{abstract}
In this paper, some potential applications of simulation in structural reliability and availability are presented. Unlike the analytical methods, where assumptions that simplify complex analysis often need to be made, simulation methods can model the structure reliability without simplifying the problem. We propose the use of statistical distributions and techniques -such as survival analysis- to model component-level reliability. Then, using failure- and repair-time distributions and information about the structural logical topology, structural reliability and availability information can be inferred through the use of discrete-event simulation techniques. Two numerical examples illustrate some potential applications of the proposed methodology.
\end{abstract}




\section{Introduction}

Structural Reliability \& Availability (R\&A) are critical issues in industrial engineering that have a daily impact on billions of people around the world, especially with the current trend towards globalization being enabled by structures such as bridges, airport towers, and government buildings. Historically, structural R\&A has focused solely on methods for assessing the safety of civil and industrial engineering projects. However, with new emerging technologies such as wind and solar energy, structural reliability has a much broader range of applications that include the design and maintenance process of such structures. Some interesting references containing attractive cases with structural reliability problems are Blischke et al. (2000), Faulin et al. (2010), and Modarres et al. (2010).

Within the scope of structural R\&A, a major concern of civil engineers is the ability to predict the lifecycle of structures that are exposed to stressful conditions such as constant workloads or natural disasters. Due to these exposures, the structures suffer degradation in the form of deterioration, deformation, fatigue, etc., resulting in possible environmental hazards. Thus, it must be noted that the state of the structures are persistently changing over time, as opposed to constant as suggested in other literature. According to $\mathrm{Li}$ (1995) there are three major ways in which structural concrete may deteriorate, namely: (1) surface deterioration of the concrete, (2) internal degradation of the concrete, and (3) corrosion of reinforcing steel in concrete. Of these, reinforcing steel corrosion is the most common form of deterioration in concrete structures and is the main target for the durability requirements pre-scribed in most design codes for concrete structures (Nilson et al., 2003). Overall, these aggressive conditions indicate that we should consider a structure's evolution over time when analyzing structural $R \& A$.

The importance of using structural R\&A to both design and maintain modern structures is highlighted by two recent examples of structural disasters, namely the Gulf Coast Oil Disaster (BP Oil Spill) and the accident in the Tibidabo (Barcelona, Spain) amusement park on July 17, 2010. The Gulf Coast Oil Disaster, regarded as one of America's worst environmental disasters, has resulted in being the largest accidental oil spill not in only the US, but in the world's history (Silverleib, 2010). The disaster was caused by an explosion on an offshore oil drilling rig that perhaps, through some careful structural R\&A analysis, could have been prevented. Similarly, the accident in the Tibidabo, which resulted in one death and two others with severe injuries was the consequence of a component failure, a possible sign of ineffective maintenance policies. 
In this paper we propose the use of non-deterministic approaches - specifically those based on discrete-event simulation (DES) - as the most natural way to deal with uncertainties in time-dependent structural reliability and availability analysis. We begin by discussing how non-deterministic approaches differ from other approaches in the field and the benefits it may bring, such as higher accuracy in determining the reliability state of structures, which can be viewed as time-dependent systems through the consideration of time-dependent components and system topology. Our DES approach is then introduced along with two examples, namely a structural reliability case and a structural availability case. These examples illustrate just a few of the many applications that the DES approach offers within the structural R\&A arena.

\section{Structural Reliability and Existing Analysis Methods}

Structural Reliability is an engineering discipline that provides a series of concepts, methods and tools to predict and/or determine the reliability, availability and safety of buildings, bridges, industrial plants, off-shore platforms and other structures, both during their design stage and during their operational lifecycle. As suggested by Melchers (1999), for any given structure, it is possible to define a set of limit states. These limit states represent varying levels of operative reliability and availability, which ranges from a fully operational state to a completely collapsed state. From a formal perspective, Structural Reliability is defined as the probability that a structure will not achieve each specified limit state -i.e. will not suffer a failure of certain type- during a specified period of time (Thoft-Christensen and Murotsu, 1986). The reliability or survival function is the probability that the structure will not have achieved the corresponding limit state at a given time. From a reliability point of view, one of the main targets of structural reliability is to provide an assembly of components which, when mounted together, will perform satisfactorily without suffering critical or relevant failures for some specified time period, either with or without maintenance policies.

In most cases, a structure can be viewed as a system of components (or individual elements) linked together by an underlying logical topology that describes the interactions and dependencies among the components. Each of these components deteriorates according to an analytical degradation or survival function and, therefore, the structural reliability is a function of each component's reliability function and the logical topology. Thus, it seems reasonable to assess the probability of failure of the structure based upon its elements' failure probability information (Mahadevan and Raghothamachar, 2000) (Coit, 2000). As described by Frangopol and Maute (2003), depending on the structure's topology, material behavior, statistical correlation, and

IN3 Working Paper Series is a monograph series promoted by the Internet Interdisciplinary Institute (IN3) of the UOC IN3 Working Paper Series (2010) | ISSN 2013-8644 | http://in3wps.uoc.edu 
variability in loads and strengths, the reliability of a structural system can be significantly different from the reliability of its components.

Therefore, the reliability of a structural system may be estimated at two levels: component level and system or structural level. At the component level, limit-state formulations, in addition to efficient analytical and simulation procedures, have been developed for reliability estimation (Park et al., 2004). In particular, a new structure will most likely have some components that have been used in other structural designs, from which there is an existing set of available data; on the other hand, if a new structure uses components about which no historical data exists, then survival analysis methods, such as accelerated life testing, can be used to obtain information about component reliability behavior (Meeker and Escobar, 1998). Structural-level analysis, on the other hand, addresses two types of issues: (1) multiple performance criteria or multiple structural states, and (2) multiple paths or sequences of individual component failures leading to overall structural failure. In structural level analysis, however, we must take into account any possible dependencies between components such as redundancy or reinforcement.

In general, structures must agree to a set of minimum design and construction standards, known as codes of practice, that correspond to the type of structure being designed. However, as noted by Lertwongkornkit et al. (2001), it is becoming increasingly common to design buildings and other civil infrastructure systems with an underlying "performance-based" objective which might consider more than just two structural states (collapsed or not collapsed); therefore, making it necessary to develop new techniques in order to account for uncertainty on key random variables affecting structural behavior. According to other authors (Marek et al., 1996) (Vukazich and Marek, 2001) standards for structural design are basically a summary of the current "state of knowledge" but offer only limited information about the real evolution of the structure through time. Therefore, these authors strongly recommend the use of probabilistic techniques, which require fewer assumptions, in order to deal with the uncertainties in structural design and decision-making such as how and when to perform maintenance on a structure. Camarinopoulos et al. (1999) do also recommend the use of probabilistic methods as a more rational approach to deal with safety problems in structural engineering. In their words, "these [probabilistic] methods provide basic tools for evaluating structural safety quantitatively". Moreover, Banks et al. (2009) emphasize the usefulness of simulation modeling for both predicting the effect of changes to existing systems and as a design tool to predict the performance of a system under varying sets of conditions.

As Park et al. (2004) suggest, it is difficult to calculate probabilities for each limitstate of a structural system. Structural reliability analysis can be performed using analytical methods or simulation-based methods (Mahadevan and Raghothamachar, 2000). On one hand, analytical methods tend to be complex and generally involve restrictive simplifying assumptions about structural behavior, which makes them difficult to apply in real scenarios. On the other hand, simulation-based methods can 
also incorporate realistic structural behavior (Billinton and Wang, 1999) (Marek et al., 1996) (Laumakis and Harlow, 2002). Traditionally, simulation-based methods have been considered to be computationally expensive, especially when dealing with highly reliable structures (Marquez, 2005). This is because when there is a low failure rate, a large number of simulations are needed in order to get accurate estimates -this is usually known as the "rare-event problem". Under these circumstances, the use of variance reduction techniques (such as importance sampling) is usually recommended. For an excellent review on simulation concepts, techniques, methods, and applications, the reader is referred to Banks et al. (2009), Law (2006), or Ross (2006). Nevertheless, in our opinion these computational concerns can now be considered mostly obsolete due to outstanding improvement in processing power experienced in recent years. This is especially true when the goal -as in our case- is to estimate time-dependent structural $R \& A$ functions, where the rare-event problem is not a major issue.

\section{A Discrete-Event Based Approach}

Consider a structure with several components which are connected together according to a known logical topology, a set of minimal paths describing combinations of components that must be operating in order to avoid a structural failure of some kind. Also assume that time-dependent reliability/availability functions are known at the component-level, i.e., each component failure- and/or repair- time distribution is known. As discussed before, this information might have been obtained from historical records or, alternatively, from survival analysis techniques -e.g. accelerated life tests- on individual components. Therefore, at any moment in time the structure will be in one of the following states: (1) perfect condition, i.e.: all components are in perfect condition and thus the structure is fully operational; (2) slight damage, i.e.: some components have experienced failures but this has not affected the structural operability in a significant way; (3) severe damage, i.e.: some components have failed and this has significantly limited the structural operability; and (4) collapsed, i.e.: some components have failed and this might imply structural collapse. Notice that, under these circumstances, there are three possible types of structural failures that can lead to a change in the state of the structure. Of course, the most relevant -and hopefully least frequent- of these structural failures is structural collapse, but sometimes it might also be interesting to be able to estimate the reliability or availability functions associated with other structural failures as well. To attain this goal, DES can be used to artificially generate a random sample of structural lifecycles, as seen in Figure 1.

IN3 Working Paper Series is a monograph series promoted by the Internet Interdisciplinary Institute (IN3) of the UOC IN3 Working Paper Series (2010) | ISSN 2013-8644 | http://in3wps.uoc.edu 




Figure 1: Using DES to generate a structural lifecycle

In effect, as explained by Faulin et al. (2008) component-level failure- and repairtime distributions can be used to randomly schedule component-level failures and repairs. Therefore, it is possible to track the current state of each individual component at each target time. This information is then combined with the structural logical topology to infer the structural state at each target time.

By repeating this process, a set of randomly generated lifecycles is provided for the given structure. Each of these lifecycles provides observations of the structural state at each target-time. Therefore, once a sufficient number of iterations have been run, accurate point and interval estimates can be calculated for the structural reliability at each target time (Juan and Vila, 2002). Also, additional information can be obtained from these runs, such as: which components are more likely to fail, which component failures are more likely to cause structural failures (failure criticality indices), which structural failures occur more frequently, etc. (Juan et al., 2007).

Moreover, notice that DES could also be employed to analyze different scenarios (what-if analysis), i.e.: to study the effects of a different logical topology on structural reliability, the effects of adding some redundant components on structural reliability, or even the effects of improving reliability of some individual components. Finally, DES also allows for considering the effect of dependencies among component failures and/or repairs. It is usually the case that a component failure or repair affects the failure or repair rate of other components. In other words, component failure- and repair-times are not independent in most real situations. Again, discrete-event simulation can handle this complexity by simply updating the failure- or repair-time distributions of each component each time a new component failure or repair takes place (Faulin et al., 2008). This way, dependencies can be also introduced in the model. Notice that this represents a major difference between our approach and other 
approaches -mainly analytical ones-, where dependencies among components, repair-times or multi-state structures are difficult to consider.

Thus, Figure 2 shows a step-by-step flowchart of our discrete-event based approach to structural reliability and availability problems.

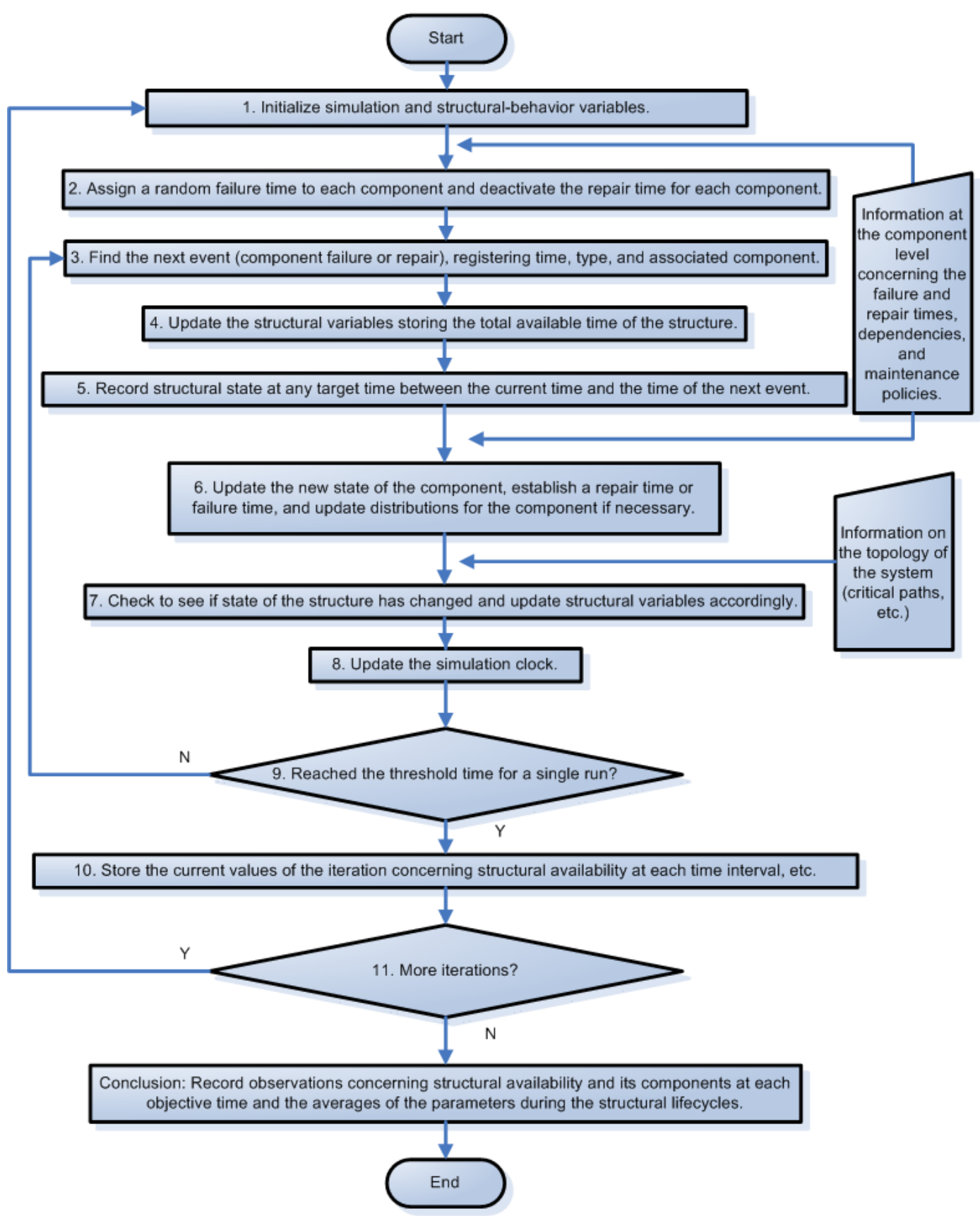

Figure 2: Discrete-Event Based Methodology for Structural R\&A Analysis

11 


\section{Experiment 1: Structural Reliability}

We present here a case study of two possible designs for a bridge. As can be seen in Figure 3 , there is an original design (Case A) and an alternative with redundant components (Case B).
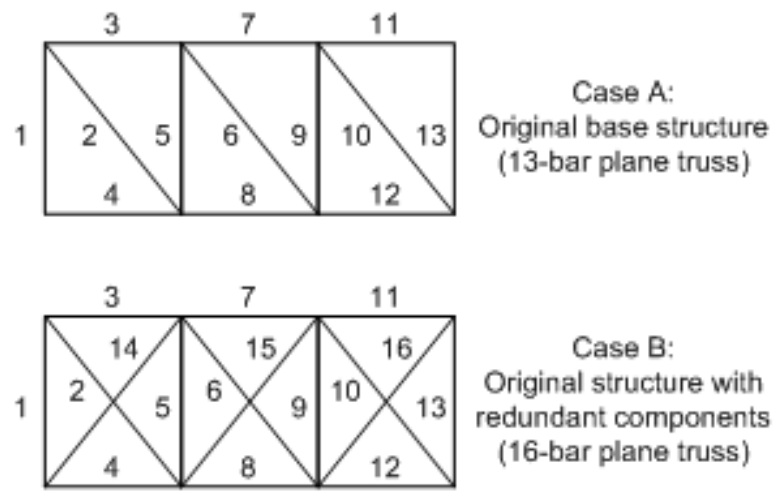

Figure 3: Different possible designs for a structure

Our first goal is to illustrate how a statistical modeling approach can be used in the design phase to help pick the most appropriate design, depending on factors such as the desired structural reliability, the available budget (cost factor) and other project restrictions. As explained before, different levels of failure can be defined for each structure, and in examining how and when the structures fail in these ways, it is possible to measure their reliability as a function of time. Different survival functions can be then obtained for a given structure, one for each structural failure type. By comparing the reliability of one bridge to another, one can determine whether a certain increase in structural robustness -either via redundancy or via reinforcement- is worthwhile according to the engineer's utility function. As can be deduced from Figure 3 , the two possible bridges are the same length and height, but the second one (case B) has 3 more trusses connecting the top and bottom beam and is thus more structurally redundant. If the trusses have the same dimensions, the second bridge should have higher reliability than the first one (case A) for a longer period of time. Regardless the failure definition for the first bridge, the second bridge will need more time to suffer from a similar failure. Analogously, a bridge with reinforced components or improved individual reliability is expected to be more reliable.

Let us consider three different types of failure. Type 1 failure corresponds to slight damage, where the structure is no longer as robust as it was at the beginning but it can still be expected to perform the function it was built for. Type 2 failures correspond to severe damage, where the structure is no longer stable but it is still standing. Finally, Type 3 failure corresponds to complete structural failure, or col-lapse. Now we have four states to describe the structure, but only two (failed or not failed) to describe each

12

IN3 Working Paper Series is a monograph series promoted by the Internet Interdisciplinary Institute (IN3) of the UOC IN3 Working Paper Series (2010) | ISSN 2013-8644 | http://in3wps.uoc.edu 
component of the structure. We can track the state of the structure by tracking the states of its components. Also, we can compare the reliabilities of the two different structures over time, taking into account that different numbers of component failures will correspond to each type of structural failure depending on the structure. For example, a component failure in Case A could lead to a Type 2 failure (severe damage), while it would only lead to a Type 1 failure (slight damage) in the Case B bridge. In other words, for Case B it will take at least two components to fail in the same section of the bridge before the structure experiences a Type 2 failure.

The first step in order to develop a numerical example will be to define the logical topology for each design. For Case A, only one minimal path must be considered since the structure will be severely damaged (the kind of "failure" we are interested in) whenever one of its components fails. However, for Case B a total of 110 minimal paths were identified. The structure will not experience a type 2 failure if, and only if, all components in any of those minimal paths are still operative (Faulin et al., 2008).

As a next step, we will allocate reliability at the component level such that the system meets its overall target. We will assume the desired reliability after 15 years is 0.90. Finding alternative designs that meet the desired target would allow the stake holders to select the most attractive (cheapest) alternative that meets the given target. Once the optimum individual component reliability is obtained for the different designs, decisions can be made regarding the individual components.

For Case A, obtaining the individual components target reliabilities (and assuming no other feasibility constrains) is a straight forward matter. A reliability block diagram (RBD) can visually display the reliability logic for a system and is widely used in survival analysis. The RBD for Case A is show in Figure 4.



Figure 4: Reliability Block Diagram for Case A

Since in Case A only one minimal path is exists, all components are equally important and therefore the individual component reliability can be obtained as:

$$
R_{i}(10 \text { years })=R_{\text {system }}(10 \text { years })^{\frac{1}{N}}
$$

Where $R_{i}$ is the individual component target reliability, $R_{\text {system }}$ is the system target reliability and $N$ is the number of components. The reliability for each individual component can then be calculated as 0.9919 .

For Case B however, the component optimum reliability cannot be obtained as easily because the criticality of the different components is not the same. The RBD for Case $B$ is show in Figure 5. 


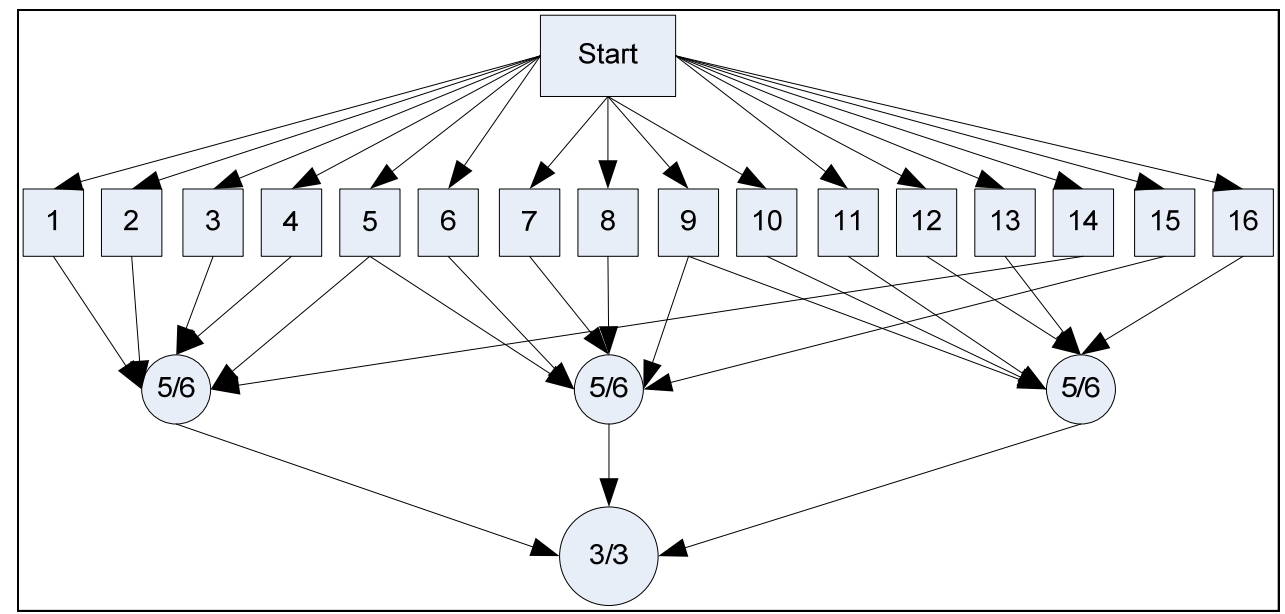

Figure 5: Reliability Block Diagram for Case B

From the RBD, the system reliability function which is function of its component reliability can be derived. Let's define the reliability importance of each component as follows (Wang et al. 2004):

$$
I_{k}(t)=\frac{\partial R_{S}(t)}{\partial R_{k}(t)}
$$

Figure 6 shows the reliability importance for the different components in Case B. Notice that components 9 and 5 are identified as the most critical components, that is, a change in the reliability of these components will have the most impact on the reliability of the system.



Figure 6: Reliability Importances for Case B

14 
In order to obtain optimum reliabilities for the components, we will use the reliability allocation method described by Mettas A. (2000). For the sake of simplicity, equal initial reliabilities, equal feasibilities and equal maximum reliabilities are assumed. The optimum component reliabilities are shown in Table 1.

Table 1: Target Reliabilities for Components in Case B

\begin{tabular}{|c|c|c|c|}
\hline Component & R(10 years) & Component & R(10 years) \\
\hline 1 & 0.9536 & 9 & 0.9269 \\
\hline 2 & 0.9536 & 10 & 0.9536 \\
\hline 3 & 0.9536 & 11 & 0.9536 \\
\hline 4 & 0.9536 & 12 & 0.9536 \\
\hline 5 & 0.9269 & 13 & 0.9536 \\
\hline 6 & 0.9505 & 14 & 0.9536 \\
\hline 7 & 0.9505 & 15 & 0.9505 \\
\hline 8 & 0.9505 & 16 & 0.9536 \\
\hline
\end{tabular}

Once the component target reliabilities are obtained, design choices regarding what components should be utilized can be made. Tables 2 and 3 contain failure-time distributions of components that would meet these requirements for designs $A$ and $B$ respectively. Figure 7 shows the survival (reliability) functions obtained in each case after a Type 2 failure-notice that similar curves could be obtained for other types of failures. This survival function shows the probability that each bridge will not have failed -according to the definition of a Type 2 failure- after some time (expressed in years). As expected, both Cases A and B meet the reliability requirement of 0.9 at 15 years. In this example, both cases are similar in reliability until the target time of 15 years after which Case B drops quicker in reliability. This is due to the fact that in order for design A to meet the reliability target, components that are much more reliable are needed, which might be a costly option. Notice that this conclusion holds only for the current values in Tables 2 and 3; should the shape and scale parameters change -e.g. by changing the quality of the components-, the survival functions would be different.

Table 2: Failure-time distributions at component level for Case A

\begin{tabular}{|c|c|r|l|c|c|r|l|}
\hline Component & Distribution & \multicolumn{1}{|c|}{ Shape } & Scale & Component & Distribution & Shape & Scale \\
\hline 1 & Weibull & 4 & 50.0 & 8 & Weibull & 4 & 50.0 \\
\hline 2 & Weibull & 4 & 50.0 & 9 & Weibull & 4 & 50.0 \\
\hline 3 & Weibull & 4 & 50.0 & 10 & Weibull & 4 & 50.0 \\
\hline 4 & Weibull & 4 & 50.0 & 11 & Weibull & 4 & 50.0 \\
\hline 5 & Weibull & 4 & 50.0 & 12 & Weibull & 4 & 50.0 \\
\hline 6 & Weibull & 4 & 50.0 & 13 & Weibull & 4 & 50.0 \\
\hline 7 & Weibull & 4 & 50.0 & - & - & - & - \\
\hline
\end{tabular}


Table 3: Failure-time distributions at component level for Case B

\begin{tabular}{|c|r|r|l|c|c|r|l|}
\hline Component & Distribution & Shape & Scale & Component & Distribution & Shape & Scale \\
\hline 1 & Weibull & 4 & 28.6 & 9 & Weibull & 4 & 32.1 \\
\hline 2 & Weibull & 4 & 32.1 & 10 & Weibull & 4 & 31.6 \\
\hline 3 & Weibull & 4 & 28.6 & 11 & Weibull & 4 & 31.6 \\
\hline 4 & Weibull & 4 & 31.6 & 12 & Weibull & 4 & 31.6 \\
\hline 5 & Weibull & 4 & 32.1 & 13 & Weibull & 4 & 32.1 \\
\hline 6 & Weibull & 4 & 32.1 & 14 & Weibull & 4 & 32.1 \\
\hline 7 & Weibull & 4 & 32.1 & 15 & Weibull & 4 & 32.1 \\
\hline 8 & Weibull & 4 & 32.1 & 16 & Weibull & 4 & 32.1 \\
\hline
\end{tabular}

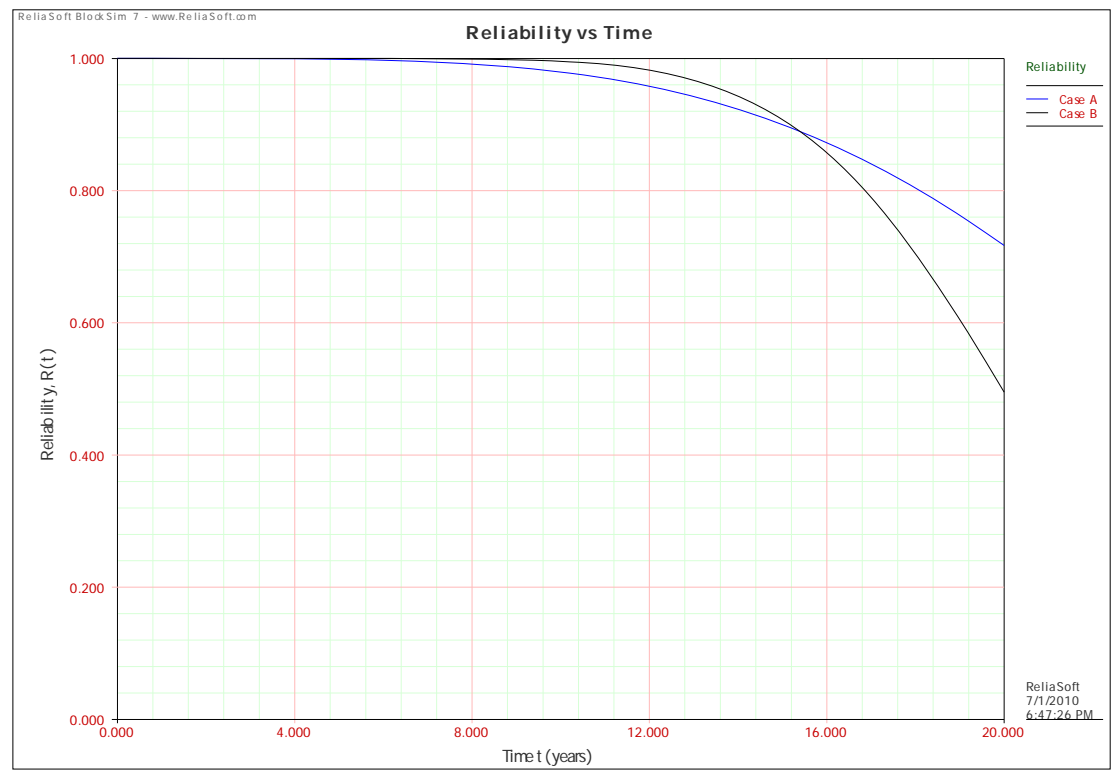

Figure 7: Survival (Reliability) functions for alternative designs

Because both alternatives have been designed to meet the same reliability target, the decision can now be made based on economic factors such as the acquisition cost of components that will meet the desired reliability. Other metrics of interest such as availability and maintenance costs of the structure would have to include additional parameters such as repair distributions, cost of maintenance, cost and frequency of inspections, efficiency of repairs, etc. DES can then be used to expand the scope of the analysis. 


\section{Experiment 2: Structural Availability}

As shown in the previous example, analytical solutions may be used in a variety of situations. However, as more complex and realistic factors need to be taken into account, analytical approaches cannot provide answers without significant assumptions that may compromise the usefulness of the results. For example, as repair distributions, efficiencies of the repairs, cost and availability of resources, dependencies between components, preventive and condition based maintenances are taken into account, analytical approaches will need to make simplifying assumptions in order to obtain mathematical models. Nevertheless, DES does not have such limitations.

With DES, one can consider the effect of maintenance policies and track the structural availability of the system, as well as the associated costs of those repairs. For the purpose of illustrating our methodology, we will assume that in the previous example, Case B is found to be more economically feasible than case A and therefore is selected as the preferable design. We will also assume that historical data is available for the estimation of repair time distributions for the trusses (Table 4). We will select three plausible maintenance policy scenarios and estimate both the availability of the bridge over a mission time of 100 years as well as the maintenance costs. Case I will assume no preventive maintenance is performed, that is, repairs will be performed when a truss failure occurs. Case II will assume preventive maintenances with a frequency of 5 years and an efficiency of the repair of $70 \%$. That is every 5 years, the structure will undergo preventive maintenance, effectively refurbishing the accumulated damage of each truss by $70 \%$. Case III will assume a condition based maintenance with biyearly inspections. An inspection that finds a truss within the last $5 \%$ of its useful life will trigger a replacement of the truss. Table 4 shows preventive maintenance time distributions. For purposes of a cost analysis, we will assume a repair upon failure of a truss costs $\$ 10,000$, a preventive maintenance will cost $\$ 1,000$, the overall cost of inspecting the bridge is $\$ 5,000$ and a truss repair triggered by an inspection discovering a critically degraded condition costs $\$ 5,000$.

Table 4: Repair-time and preventive maintenance time distributions at component level

\begin{tabular}{|c|c|r|r|r|r|c|c|}
\hline \multicolumn{4}{|c|}{ Repair-time } & \multicolumn{4}{c|}{ Preventive maintenance time } \\
\hline Component & Distribution & \multicolumn{1}{|c|}{ Shape } & \multicolumn{1}{l|}{ Scale } & Component & Distribution & Shape & Scale \\
\hline 1 & Weibull & 2 & 0.5 & 1 & Weibull & 2 & 0.3 \\
\hline 2 & Weibull & 1.8 & 0.5 & 2 & Weibull & 1.8 & 0.3 \\
\hline 3 & Weibull & 1.8 & 0.3 & 3 & Weibull & 1.8 & 0.2 \\
\hline 4 & Weibull & 2 & 0.3 & 4 & Weibull & 2 & 0.2 \\
\hline 5 & Weibull & 2 & 0.5 & 5 & Weibull & 2 & 0.3 \\
\hline 6 & Weibull & 1.8 & 0.5 & 6 & Weibull & 1.8 & 0.3 \\
\hline
\end{tabular}

17 


\begin{tabular}{|c|c|c|c|c|c|c|c|}
\hline 7 & Weibull & 1.8 & 0.3 & 7 & Weibull & 1.8 & 0.2 \\
\hline 8 & Weibull & 1.8 & 0.3 & 8 & Weibull & 1.8 & 0.2 \\
\hline 9 & Weibull & 2 & 0.5 & 9 & Weibull & 2 & 0.3 \\
\hline 10 & Weibull & 1.8 & 0.5 & 10 & Weibull & 1.8 & 0.3 \\
\hline 11 & Weibull & 1.8 & 0.3 & 11 & Weibull & 1.8 & 0.2 \\
\hline 12 & Weibull & 1.8 & 0.3 & 12 & Weibull & 1.8 & 0.2 \\
\hline 13 & Weibull & 2 & 0.5 & 13 & Weibull & 2 & 0.3 \\
\hline 14 & Weibull & 1.8 & 0.5 & 14 & Weibull & 1.8 & 0.3 \\
\hline 15 & Weibull & 1.8 & 0.5 & 15 & Weibull & 1.8 & 0.3 \\
\hline 16 & Weibull & 1.8 & 0.5 & 16 & Weibull & 1.8 & 0.3 \\
\hline
\end{tabular}

Using the proposed DES approach, the structural availability over time, specifically the probability that the structure under each scenario will be operative - not suffering a Type 2 or Type 3 failure - at any given time, can be obtained. Figure 8 shows the availability functions obtained for each alternative maintenance policy over a mission time of 100 years. Figure 9 and 10 show the expected costs and system failures over time for each alternative. Notice that the costs in this analysis do not include the cost of a system failure which is an important factor, particularly where safety is at risk. The expected number of system failures can be used to factor in this additional cost. From expected costs and number of system failures it can be concluded that the interval preventive maintenance policy is most desirable in the first 40 years of the life of the bridge. As the bridge ages, a more aggressive policy such as regular inspections of the condition of the bridge seem to be more efficient. Note that these conclusions hold for the assumed inputs (e.g. failure and repair distributions, repair efficiencies, maintenance frequencies, etc.). Alternative inputs may lead to different conclusions.

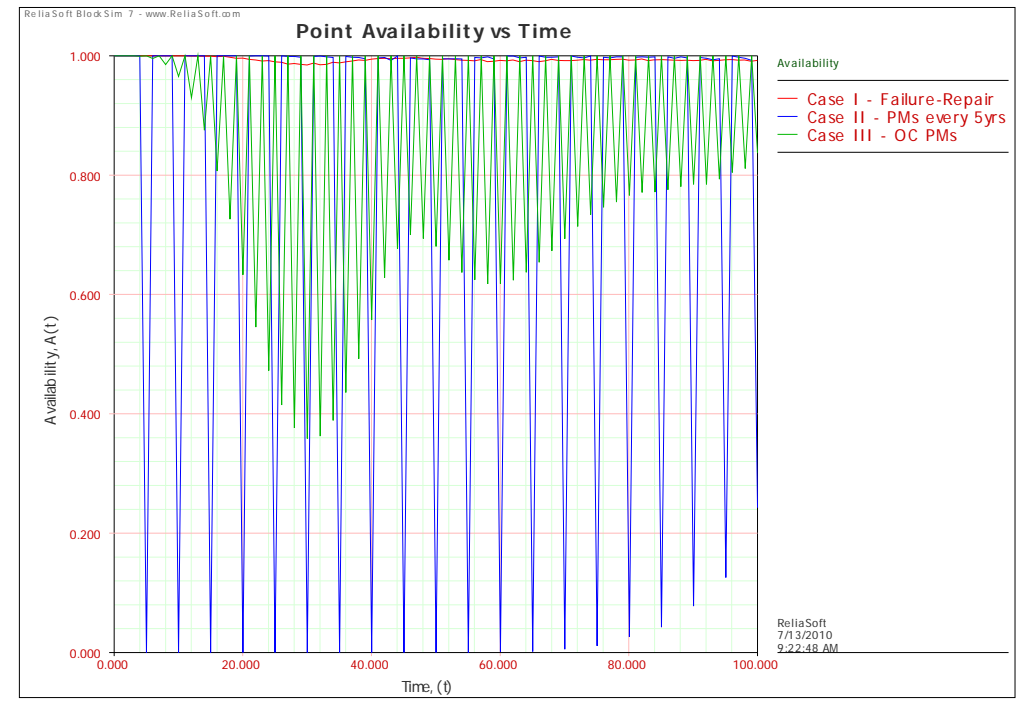

Figure 8: Availability function for alternative maintenance policies 




Figure 9: Expected number of Type 2 or 3 failures for alternative maintenance policies



Figure 10: Expected cost for alternative maintenance policies

Figure 11 shows in more detail the simulated lifetime of the structure under the conditions in Case I, notice how the availability score $A(t)$ fluctuates over time, depending on the time length since the previous maintenance. See Appendix for the table of values for Case II and III. 
Point Results at Preselected System Times

\begin{tabular}{|c|c|c|c|c|c|c|c|c|c|c|c|c|c|c|c|}
\hline \multicolumn{16}{|c|}{ Point Results at Preselected System Times } \\
\hline 2 & 1 & 4 & 0 & 27 & 0.986 & 66920 & 0.4009 & 52 & 0.9951 & 210396 & 1.4858 & 77 & 0.9932 & 352874 & 2.5117 \\
\hline 4 & 1 & 52 & 0 & 29 & 0.9857 & 82488 & 0.5479 & 54 & 0.9926 & 220941 & 1.556 & 79 & 0.9943 & 363199 & 2.5758 \\
\hline 5 & 1 & 115 & 0 & 30 & 0.9844 & 90359 & 0.6219 & 55 & 0.9923 & 226560 & 1.5977 & 80 & 0.9924 & 368478 & 2.6119 \\
\hline 6 & 1 & 229 & 0 & 31 & 0.9876 & 98170 & 0.6929 & 56 & 0.9916 & 232192 & 1.6373 & 81 & 0.9929 & 373819 & 2.6467 \\
\hline 9 & 1 & 1063 & 0.0003 & 34 & 0.9889 & 120762 & 0.9021 & 59 & 0.9909 & 249795 & 1.7687 & 84 & 0.9928 & 389637 & 2.7507 \\
\hline 10 & 1 & 1615 & 0.0008 & 35 & 0.9881 & 127770 & 0.969 & 60 & 0.9922 & 255914 & 1.8123 & 85 & 0.9936 & 395023 & 2.7863 \\
\hline 11 & \begin{tabular}{|l|}
0.9996 \\
\end{tabular} & 2371 & 0.0016 & 36 & 0.9898 & 134347 & 1.0221 & 61 & 0.9914 & 262245 & 1.862 & 86 & 0.9934 & 400422 & 2.8221 \\
\hline 12 & 0.9999 & 3364 & 0.0023 & 37 & 0.9911 & 140618 & 1.069 & 62 & 0.9929 & 268442 & 1.9088 & 87 & 0.9923 & 405901 & 2.8624 \\
\hline 13 & 0.9991 & 4715 & 0.0044 & 38 & 0.9928 & 146516 & 1.1152 & 63 & 0.9901 & 274533 & 1.9549 & 88 & 0.9925 & 411390 & 2.8998 \\
\hline 18 & 0.9975 & 16506 & 0.0409 & 43 & 0.9948 & 170761 & 1.2643 & 68 & 0.9939 & 304269 & 2.1744 & 93 & 0.9916 & 439535 & 3.1082 \\
\hline 19 & 0.9957 & 20102 & 0.056 & 44 & 0.9955 & 175012 & 1.2867 & 69 & 0.9923 & 309931 & 2.2122 & 94 & 0.9923 & 445063 & 3.148 \\
\hline 20 & 0.996 & 24184 & 0.0767 & 45 & 0.9958 & 179136 & 1.3081 & 70 & 0.992 & 315458 & 2.2534 & 95 & 0.9932 & 450742 & 3.1848 \\
\hline 21 & 0.994 & 28946 & 0.1022 & 46 & 0.9962 & 183302 & 1.3288 & 71 & 0.9919 & 320996 & 2.2946 & 96 & 0.9935 & 456350 & 3.2242 \\
\hline 22 & 0.9929 & 34082 & 0.1353 & 47 & 0.9973 & 187477 & 1.3496 & 72 & $\begin{array}{l}0.9925 \\
\end{array}$ & 326469 & 2.3363 & 97 & 0.9925 & 461923 & 3.2653 \\
\hline 23 & 0.9913 & 39765 & 0.175 & 48 & 0.9956 & 191759 & 1.3745 & 73 & 0.9936 & 331707 & 2.3685 & 98 & 0.993 & 467556 & 3.303 \\
\hline 24 & 0.9919 & 45968 & 0.2231 & 49 & 0.9953 & 196180 & 1.4008 & 74 & 0.9927 & 337150 & 2.4067 & 99 & 0.9907 & 473256 & 3.3453 \\
\hline 25 & 0.9899 & 52643 & 0.2757 & 50 & 0.9944 & 200769 & 1.4292 & 75 & 0.9938 & 342395 & 2.4446 & 100 & 0.9923 & 478717 & 3.3858 \\
\hline
\end{tabular}

Figure 11: Resulting Values for Case I

Even though we have analyzed only the above three different maintenance policies using simulation, it is clear that DES is a useful tool to conduct a variety of analysis for real world scenarios. The results from similar analysis using simulation can then be used by decision makers to make the right choice in terms of safety, availability and financial considerations.

\section{Conclusions}

The advantages of using probabilistic methods to estimate reliability, availability and other metrics of interest in time-dependent building and civil engineering structures has been discussed. Among the available methods, discrete-event simulation (DES) seems to be the most realistic choice. DES offers clear advantages over other approaches, namely: (1) the opportunity of creating models which accurately reflect the structure's characteristics and behavior -including possible dependences among components' failure and repair times-, and (2) the possibility of obtaining additional information about the system's internal functioning and about its critical components. Therefore, a simulation-based approach is recommended for practical purposes, since 
it can consider details such as multi-state structures, dependencies among failure and repair-times, or non-perfect maintenance policies. The numerical examples discussed in this paper provide some insight on how DES can be used to estimate structural reliability and availability functions when analytical methods are not available, how it can contribute to detect critical components in a structure that should be reinforced or improved, and how to make better design decisions that consider not only the construction of such structures but also possible maintainability policies.

\section{Acknowledgments}

This work has been partially supported by the IN3-UOC Knowledge Community Program (HAROSA KC). We would also like to thank ReliaSoft Corporation for providing us with licenses of BlockSim to run our tests.

\section{References}

Banks, J., Nelson, B. Nicol, D. and Carson II, J. 2009. Discrete-Event System Simulation. Prentice Hall, Upper Saddle River, NJ, USA.

Billinton, R. and P. Wang. 1999. Teaching distribution systems reliability evaluation using Monte Carlo simulation. IEEE Transactions on Power Systems 14:397-403.

Blischke, W.R. and D.N., Prabhakar Murthy 2000. Reliability. Modeling, Prediction, and Optimization. Wiley. New York.

Camarinopoulos, L., A. Chatzoulis, M. Frondistou-Yannas and V. Kallidromitis. 1999. Assessment of the time-dependent structural reliability of buried water mains. Reliability Engineering and Safety 65(1):41-53.

Coit, D. 2000. System Reliability Prediction Priorization Strategy. In 2000 Proceedings Annual Reliability and Maintainability Symposium 175-180. Los Angeles, CA, USA.

Faulin, J., A. Juan, S. Martorell, and J.E. Ramirez-Marquez. 2010. Simulation Methods for Reliability and Availability of Complex Systems. Springer. London.

Faulin, J., A. Juan, C. Serrat and V. Bargueño. 2008. Improving Availability of TimeDependent Complex Systems by using the SAEDES Simulation Algorithms. Reliability Engineering and System Safety 93(11):1761-1771.

Frangopol, D. and K. Maute. 2003. Life-cycle reliability-based optimization of civil and aerospace structures. Computers \& Structures 81(7):397-410.

Juan, A., J. Faulin, M. Sorroche and J. Marques. 2007. J-SAEDES: A Simulation Software to improve Reliability and Availability of Computer Systems and Networks. In Proceedings of the 2007 Winter Simulation Conference, eds. S. G. Henderson, B. 
Biller, M.-H Hsieh, J. Shortle, J. D. Tew, and R. R. Barton, 1977-1985. Piscataway, New Jersey: Institute of Electrical and Electronics Engineers, Inc.

Juan, A. and A. Vila. 2002. SREMS: System Reliability Using Monte Carlo Simulation with VBA and Excel. Quality Engineering 15(2):333-340

Laumakis, P. and G. Harlow. 2002. Structural Reliability and Monte Carlo Simulation. International Journal of Mathematical Education in Science and Technology 33(3):377-387.

Law, A. 2006. Simulation Modeling and Analysis. Mcgraw Hill Higher Education.

Lertwongkornkit, P., H. Chung and L. Manuel. 2001. The Use of Computer Applications for Teaching Structural Reliability. In Proceedings of the 2001 ASEE GulfSouthwest Section Annual Conference. Austin, Texas, USA.

$\mathrm{Li}, \mathrm{C} .1995$. Computation of the Failure Probability of Deteriorating Structural Systems. Computers \& Structures 56(6):1073-1079.

Mahadevan, S. and P. Raghothamachar. 2000. Adaptive simulation for system reliability analysis of large structures. Computers \& Structures 77:725-734.

Marek P., M. Gustar and T. Anagnos. 1996. Simulation Based Reliability Assessment for Structural Engineers. CRC Press, Boca Raton, FL, USA.

Marquez, A., A. Sanchez and B. lung. 2005. Monte Carlo-Based Assessment of System Availability. A Case Study for Cogeneration Plants. Reliability Engineering \& System Safety 88(3):273-289.

Meeker, W. and L. Escobar. 1998. Statistical Methods for Reliability Data. John Wiley \& Sons.

Melchers, R. 1999. Structural Reliability: analysis and prediction. John Wiley \& Sons.

Mettas, A. 2000. Reliability Allocation and Optimization for Complex Systems. In 2000 Proceedings Annual Reliability and Maintainability Symposium. Los Angeles, CA, USA: 216-221.

Modarres, M., M. Kaminskiy, and V. Krivtsov. 2010. Reliability Engineering and Risk Analysis. A Practical Guide. CRC Press. Boca Raton, FL.

Nilson, A., D. Darwin and C. Dolan. 2003. Design of Concrete Structures. McGraw-Hill Science.

Park, S., S. Choi, C. Sikorsky and N. Stubbs. 2004. Efficient method for calculation of system reliability of a complex structure. Int. J. of Solids and Structures 41:50355050.

Ross, S. 2006. Simulation. Academic Press.

Silverleib, A. 2010. The Gulf spill: America's worst environmental disaster? CNN: http://edition.cnn.com/2010/US/08/05/gulf.worst.disaster/

Thoft-Christensen, P. and Y. Murotsu. 1986. Application of Structural Systems Reliability Theory. Springer Verlag.

Vukazich, S. and P. Marek. 2001. Structural Design Using Simulation Based Reliability Assessment. Acta Polytechnica 41(4-5):85-92. 
Wang, W., Loman, J. and Vassiliou, P. 2004. Reliability Importance of Components in a Complex Systems. In 2004 Proceedings Annual Reliability and Maintainability Symposium - RAMS. Los Angeles, CA, USA: 6-11.

\section{Author Biographies}

ANGEL A. JUAN is an Associate Professor of Simulation and Data Analysis in the Computer Science Department at the Open University of Catalonia (Barcelona, Spain), as well as a Researcher at the Internet Interdisciplinary Institute (IN3). He holds a Ph.D. in Industrial Engineering, an M.S. in Information Technologies, and a M.S. in Applied Mathematics. His research interests include computer simulation, educational data analysis and mathematical e-learning. He is an editorial board member of the Int. J. of Data Analysis Techniques and Strategies and the Int. J. of Information Systems \& Social Change. He is also a member of the INFORMS society. His webpage and email address are <http://ajuanp.wordpress.com> and <ajuanp@gmail.com>.

ARAI MONTEFORTE is the Manager of the Simulation Group at ReliaSoft Corporation. Over the years she has played a key role in the design and development of ReliaSoft's software, including extensive involvement in the BlockSim and RENO product families. Ms. Monteforte holds an M.S. degree in Reliability and Quality Engineering, a B.S. in Chemical Engineering and a B.S. in Computer Science, all from the University of Arizona. Her areas of research and interest include Stochastic Event Simulation, Design of Experiments (DOE) and System Reliability and Maintainability Analysis. She can be contacted by e-mail at <Arai.Monteforte@ReliaSoft.com>.

JAVIER FAULIN is an Associate Professor of Operations Research and Statistics at the Public University of Navarre (Pamplona, Spain). He holds a PhD in Economics, a MS in Operations Management, Logistics and Transportation and a MS in Applied Mathematics. His research interests include logistics, vehicle routing problems and simulation modeling and analysis. He is a member of INFORMS and EURO societies and an editorial board member of the International Journal of Applied Management Science and the International Journal of Operational Research and Information Systems. His e-mail address is <javier.faulin@unavarra.es>.

HUAIRUI GUO is the Director of the Theoretical Development Group at ReliaSoft Corporation. He received his Ph.D. in Systems \& Industrial Engineering and M.S. in Reliability \& Quality engineering; both from the University of Arizona. He also received his M.S. in Manufacturing Engineering from the National University of Singapore and B.S. from Xi'an Jiaotong University, China. His research and publications cover 
reliability areas, such as life data analysis, repairable system modeling and reliability test planning, and quality areas, such as process monitoring, analysis of variance and design of experiments. He is involved in the development of ReliaSoft's Weibull++, ALTA and RGA software. His e-mail is <Harry.Guo@ReliaSoft.com>.

JORGE SIMOSA is a student in Electrical Engineering and Computer Science at Massachusetts Institute of Technology (Cambridge, MA, USA). His academic curriculum ranges from Electrical Engineering topics such as Circuit Design to Computer Science topics such as Software Engineering. His research interests include Networks, Reliability \& Availability issues, and mathematical e-learning. He is currently a Junior Researcher at the Open University of Catalonia (Spain) and the Public University of Navarre (Spain), within the HAROSA Community, completing research projects concerning Structural Reliability \& Availability and e-Learning. His e-mail address is <jdsimosa@mit.edu>.

\section{Appendix}

\begin{tabular}{|c|c|c|c|c|c|c|c|c|c|c|c|c|c|c|c|}
\hline \multicolumn{16}{|c|}{ Point Results at Preselected System Times } \\
\hline Time & $A(t)$ & $\operatorname{Cost}(t)$ & Failures(t) & Time & $A(t)$ & $\cos t(t)$ & Failures(t) & Time & $A(t)$ & $\cos t(t)$ & Failures(t) & Time & $A(t)$ & $\operatorname{Cost}(t)$ & Failures(t \\
\hline 1.001 & 1 & 0 & 0 & 26.026 & 0.9993 & 91844.7 & 0.0198 & 51.051 & 0.9948 & 258815.8 & 0.4741 & 76.076 & 0.9986 & 426276.4 & 0.916 \\
\hline 2.002 & 1 & 5 & 0 & 27.027 & 0.9992 & 93338.7 & 023 & 52.052 & 0.9966 & 263169.8 & 4976 & 77.077 & 0.998 & 428847.4 & 0.9247 \\
\hline 3.003 & 1 & 20 & 0 & 28.028 & 0.999 & 95210.7 & 0279 & & 0.9945 & 267808.8 & 5279 & 78.078 & 0.9981 & 431701.4 & 0.936 \\
\hline 4.004 & 1 & 47 & 0 & 29.029 & 9986 & 97488.7 & 0351 & .054 & 0.9944 & 272879.8 & 5587 & 79.079 & 0.9975 & 434824.4 & 9486 \\
\hline 5.005 & - & 6094.5 & 0 & 30.03 & 0 & 115990.5 & 0435 & 55.055 & 0.0002 & 293555.5 & 0.59 & 80.08 & 0.0238 & 453833.2 & .9629 \\
\hline 6.006 & 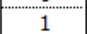 & 16108.5 & 0001 & 31.031 & 9985 & 117801.5 & 0518 & 6.056 & 0.9965 & 297154.5 & 0.6136 & 81.081 & 0.9985 & 456184.2 & .9742 \\
\hline 7.007 & 1 & 16147.5 & 0001 & 32.032 & 0.9987 & 120163.5 & .0588 & 57.057 & 0.9958 & 301248.5 & 0.6361 & 82.082 & 0.9978 & 458951.2 & .9848 \\
\hline 8.008 & 1 & 16221.5 & 001 & 33.033 & 0.9992 & 122965.5 & 677 & 58.058 & 0.9957 & 305530.5 & & 83.083 & 0.9973 & 461992.2 & 963 \\
\hline 9.009 & 1 & 16339.5 & 001 & 34.034 & 0.9981 & 126214.5 & 0.079 & 59.059 & 0.9961 & 310 & 91 & 84.084 & 0.9973 & 465285.2 & \\
\hline 10.01 & 0 & 32529.1 & 002 & 35.035 & 0 & 145603.8 & 56 & 60.06 & 0.0005 & 330 & 35 & 85.085 & 0.0486 & 484428.3 & 74 \\
\hline 11.011 & 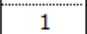 & & & 36.036 & 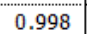 & & & & & & & & & & \\
\hline 12.012 & 1 & & & & 0.9977 & & & & & & & & 0.9977 & & \\
\hline 13.013 & 1 & 1.1 & & 38.038 & 0.9966 & 5.8 & & & 0.9973 & & & 88.088 & 0.9968 & 493296.3 & \\
\hline 14.014 & 1 & 33436.1 & 007 & 39.039 & 0.9958 & 1.8 & 81 & 4 & 0.997 & 344 & 0.7715 & 89.089 & 0.9964 & 497008.3 & 338 \\
\hline 15.015 &  & 39.8 & 11 & 40.04 & 0 & 179 & 83 & 65 & 0.0022 & 363 & 75 & 90.09 & 0.0819 & 516 & \\
\hline 16.016 & 1 & 50185.8 & 015 & 41.041 & 0.9962 & 182427.5 & 41 & 66.066 & 0.9977 & 366 & 0.8006 & 91.091 & 0.9972 & 519435.9 & 1.1201 \\
\hline 17.017 & 1 & 50631.8 & 0.0017 & 42.042 & 0.9967 & 186303.5 & 0.2221 & 67.067 & 0.9979 & 369243.9 & 0.8109 & 92.092 & 0.9967 & 522624.9 & 1.1343 \\
\hline 18.018 & . 999 & 51232.8 & 0.0022 & 43.043 & 0.994 & 190677.5 & 0.2445 & 68.068 & 0.9969 & 372327.9 & 0.8241 & 93.093 & 0.9959 & 526130.9 & .1489 \\
\hline 19.019 & 0.9998 & 52029.8 & 0.003 & 44.044 & 0.9952 & 195547.5 & 0.271 & 69.069 & 0.9973 & 375466.9 & 0.8383 & 94.094 & 0.9948 & 530126.9 & .1695 \\
\hline 20.02 & 0 & 69011.6 & 0041 & 45.045 & 0 & 216254.5 & 3024 & 70.07 & 0.0048 & 394518.9 & 0.8503 & 95.095 & 0.1206 & 549774.6 & \\
\hline 21.021 & & & & & & & & & & & & & & & \\
\hline 22.022 & 9995 & 70542.6 & & 47.047 & 0.9947 & 224 & & 72.072 & 0.9978 & 399 & & 97.097 & & 556 & \\
\hline 23.023 & 0.9999 & 71748.6 & 097 & 48.048 & 0.9947 & 229076.5 & 0.3774 & 73.073 & 0.9981 & 402249.9 & & 98.098 & 0.9952 & 560231.6 & \\
\hline 24.024 & 0.9992 & 73161.6 & 0.0129 & 49.049 & 0.9918 & 234178.5 & 0.413 & 74.074 & 0.9978 & 405205.9 & 0.8953 & 99.099 & 0.9957 & 564241.6 & 1.2642 \\
\hline 25.025 & 0 & 90783.7 & 0.0157 & 50.05 & 0.0001 & 255029.8 & 0.4431 & 75.075 & 0.0136 & 424082.4 & 0.9064 & 100.1 & 0.1593 & 583962.2 & 1.2874 \\
\hline
\end{tabular}

Figure 12: Resulting Values for Case II 


\begin{tabular}{|c|c|c|c|c|c|c|c|c|c|c|c|c|c|c|c|}
\hline Time & $A(t)$ & $\cos t(t)$ & Failures $(\mathrm{t})$ & Time & $A(t)$ & $\operatorname{Cost}(t)$ & Failures $(\mathrm{t})$ & Time & $A(t)$ & $\operatorname{Cos} t(t)$ & Failures $(\mathrm{t})$ & Time & $A(t)$ & $\operatorname{Cos} t(t)$ & Failures $(\mathrm{t})$ \\
\hline 1.001 & 1 & 0.1 & 0 & 5.026 & 41473 & 1840.678 & 012317 & 51 & 9994 & 6775.389 & 0.25287 & 76.076 & 0.74587 & 415098.924 & 0.39984 \\
\hline 002 & 99999 & 02.899 & 0 & 027 & 1 & 78 & 18 & 52 & 55737 & 8407.007 & 26648 & 77.077 & 99996 & 415360.824 & 39995 \\
\hline 003 & 1 & 2.599 & 0 & 028 & 37634 & 26985.011 & 137 & 53 & 99996 & 8623.207 & 26653 & 78.078 & 75467 & 26650.03 & 916 \\
\hline 004 & 99914 & 3.903 & 0 & .029 & 1 & 27064.111 & 139 & .054 & 3688 & 30791.545 & 28129 & 79.079 & 99988 & 426912.93 & \\
\hline 5.005 & 1 & 96.503 & 0 & 30.03 & 35857 & 1969.015 & 7445 & .055 & 99999 & 30984.045 & 0.28135 & 80.08 & 0.76582 & 438253.278 & .41896 \\
\hline 6.006 & 99529 & 1625.265 & 00002 & 1.031 & 1 & +2078.615 & 1745 & 5.056 & 0.62465 & 293473.428 & 0.29607 & 81.081 & 0.99992 & 438510.578 & 1911 \\
\hline 7.007 & 99998 & 14759.465 & 0.00008 & 32.032 & 3626 & 56346.734 & 0.19118 & 7.057 & 0.99996 & 293668.828 & 0.29613 & 82.082 & 0.77064 & 450011.973 & 0.42941 \\
\hline 8.008 & 28529 & 9913.687 & 0.00013 & 33.033 & .99998 & 56480.334 & 126 & 8.058 & 0.61767 & 306305.094 & 28 & 83.083 & 0.9999 & 450263.473 & 0.42953 \\
\hline 9.009 & 89996 & 0169.487 & 0002 & 34.034 & 38899 & 226 & 18 & 59.059 & 0.99998 & 694 & 37 & 84.084 & 0.7715 & .257 & 1072 \\
\hline 10.01 & 96513 & 25703.262 & 00 & 35.035 & 99997 & & & 60.06 & 787 & 762 & & 85.085 & 0.99997 & 462 & \\
\hline 011 & 99982 & 5093 & 0.001 & 36.036 & 43556 & 07 & 66 & 1.061 & 0.99997 & +62 & & 86.086 & 0.77543 & 473 & 249 \\
\hline .012 & 2967 & 32241.186 & 00247 & 37.037 & 99995 & 567 & 74 & 2.062 & 0.62383 & 963 & 41 & 87.087 & 0.99989 & 474 & 266 \\
\hline 13.013 & 99977 & 32745.886 & 00288 & 38.038 & 49218 & 939 & 99 & 3.063 & 0.99993 & 6.063 & 554 & 88.088 & 0.78068 & 4860 & 0.46396 \\
\hline 14.014 & 87516 & 39756.65 & 0.00581 & 39.039 & 99995 & 739 & 107 & 4.064 & 0.63711 & 4.244 & 709 & 89.089 & 0.99993 & 486252.024 & 0.4641 \\
\hline 15.015 & 99965 & 333.65 & 0.00626 & 40.04 & 55707 & 395 & 381 & 5.065 & 0.99991 & 044 & 721 & 90.09 & 0.78461 & 4981 & .47541 \\
\hline 16.016 & 80691 & 48500.645 & 0.01287 & 1.041 & 0.99994 & .395 & 389 & 66.066 & 0.65393 & 938 & 16 & 91.091 & .99992 & 4984 & 551 \\
\hline 17.017 & 99968 & 48998.445 & 0.01324 & 42.042 & 0.62792 & 13974.679 & 0.21746 & 67.067 & 0.99995 & 35693 & 726 & 92.092 & 0.78456 & 510367.861 & 0.48642 \\
\hline 18.018 & 72601 & 58542.485 & 02384 & 43.043 & 0.99993 & 379 & 76 & 68.068 & 0.67318 & 27 & 01 & 93.093 & .99995 & 510 & 0.48652 \\
\hline 19.019 & 99979 & 588 & 05 & 4.044 & 0.67615 & 224 & 26 & 69.069 & 0.99997 & 57 & & 94.094 & 1.79307 & 522526.504 & 0.49738 \\
\hline 20.02 & 63303 & 700 & & 45.045 & 0.9 & & & 77 & & 244 & & 095 & 994 & 522 & \\
\hline 21.021 & 1 & 700 & & 46.046 & 0.69 & 23 & & 71.071 & 0.9 & 44 & & 96.096 & 0.80421 & 534 & 86 \\
\hline 22.022 & 54531 & 829 & & $4 \%$ & 0.99989 & 2346 & & 72 . & 0.7 & 667 & & 97.097 & 0.99994 & 85 & 99 \\
\hline 23.023 & 99999 & 8301 & & 48.048 & .6935 & & & 73.073 & 0.9 & 367 & & 98.098 & 0.81085 & 546 & 0.51806 \\
\hline 24.024 & 47219 & & & 49.0 & & & & & & & & 99.099 & 0.5 & 546 & 0.5 \\
\hline 25.025 & 1 & 97059.009 & 0.09365 & 50.05 & 0.68028 & 256539.189 & 0.2 & 75.075 & 0.99989 & 3843.638 & 0.39114 & 100.1 & 0.82007 & 558656.756 & 39 \\
\hline
\end{tabular}

Figure 13: Resulting Values for Case III 


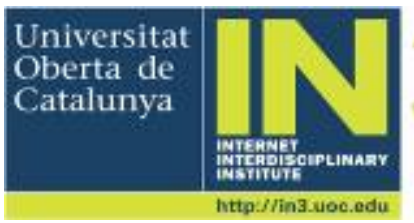

